\title{
Effects of Lycopene Alone or Combined with Melatonin on Methotrexate-Induced Nephrotoxicity in Rats
}

\author{
Elif Oguz ${ }^{1 *}$, Sezen Kocarslan², Suzan Tabur ${ }^{3}$, Hatice Sezen ${ }^{4}$, Zehra Yilmaz ${ }^{1}$, \\ Nurten Aksoy ${ }^{4}$
}

\begin{abstract}
Methotrexate (Mtx), used for its anticancer and immunsuppresive properties, is known to be a nephrotoxic agent. We aimed to investigate the effects of lycopene (Lyc) alone or combined with melatonin (Mel) on Mtxinduced nephrotoxicity since both of these agents have antioxidant and anti-inflammatory effects. Nephrotoxicity was induced by intraperitoneal administration of methotrexate at a dose of $20 \mathrm{mg} / \mathrm{kg}$. Treatment both with Lyc alone and Lyc combined with Mel provided significant reduction in tumor necrosis factor-alpha, interleukin 1-beta and ceruloplasmin levels in Mtx administered rats. Hovewer, Lyc combined with Mel provided a significant reduction also in NO levels. Hstopathological examination showed that there was an obvious improvement in the degenerative changes compared to Mtx administrated group with the Lyc combined Mel group giving best protection. In conclusion Lyc alone and combined with Mel provided significant improvement against renal damage caused by Mtx, preseumably via antioxidant and anti-inflammatory activities.
\end{abstract}

Keywords: Lycopene - melatonin - methotrexate - nephrotoxicity

Asian Pac J Cancer Prev, 16 (14), 6061-6066

\section{Introduction}

Methotrexate (Mtx), an antifolate, is used as an anti-cancer drug in higher doses such as lymphoma, breast carcinoma, sarcoma, breast cancer (Choudhury et al., 2000; Miyazaki et al., 2003; Seigers et al., 2008; Findlay et al., 2008; D'Adamo, 2011) and also in lower doses in the treatment of rheumatic diseases, psoriasis, refractory inflammatory bowel disease since it has immunosuppressant effect (Peters et al., 2000; Gisondi and Girolomoni, 2007; Renna, 2014).

However, nephrotoxicity, a native adverse effect of certain anticancer drugs, is also one of the complications of Mtx therapy (Asvadi et al., 2011; Lameire et al., 2011). It has been suggested that oxidative stress and inflammation may have role inthe pathogenesis of MTX-induced nephrotoxicity rather than blockage in folate synthesis in kidneys (Devrim et al., 2005; Asvadi et al., 2011).

Lyc (Lyc) is a natural pigment, presented in tomato and other red fruits and vegetables such as watermelon, pink grape fruit (Clinton, 1998). It has many biochemical functions as a highly efficient antioxidant and free radical scavenging agent and inhibitor of proinflamatory and pro-thrombotic factors (Cohen, 2002; Tapiero et al., 2004; Velmurugan et al., 2004; Mordente et al., 2011). It is twice as effective as $\beta$-carotene and 10-fold more activethan $\alpha$-tocopherol as an antioxidant (Di Mascio, 1989) that provides protectionagainst cellular damage caused by reactive oxygen species.

It was reported that Lyc has a protective effect against development of diabetic nephropathy and ameliorates renal function via improving oxidative status (Li et al., 2014). Koul et al. (2013) found that Lyc may be beneficial in mitigating doxorubicin induced nephrotoxicity in mice. Protective effects of Lyc have been shown against cisplatin and cyclosporine -induced nephrotoxicity and oxidative stress in rats (Atessahin et al., 2005; Atessahin et al., 2007). These antioxidant and anti-inflammatory effects of Lyc make us think that it may have benefical effects on Mtx-induced nephrotoxicity.

Mel has been shown to have free radical scavenging actions at both physiologic and pharmacologic concentrations. A number of studies have shown that $\mathrm{Mel}$ is significantly better than the classic antioxidants in resisting free radical-induced molecular destruction (Gultekin et al., 2001; Baydas et al., 2002). Beneficial antioxidant effects of Mel have been recently shown in clinical settings for several chronic diseases, including patients with rheumatoid arthritis (Forrest et al., 2007), elderly patients with primary essential hypertension (KedzioraKornatowska, 2008), and females with infertility. Several experimental studies reveal the benefical effect of Mel on renal damage. Chronic Mel treatment reduces renal injury by reducing lipid oxidation and NO production in

${ }^{1}$ Department of Medical Pharmacology, ${ }^{2}$ Department of Medical Pathology, ${ }^{4}$ Department of Medical Biochemistry, Faculty of Medicine, Harran University, Sanliurfa, ${ }^{3}$ Department of Endocrinology, Faculty of Medicine, Gaziantep University, Gaziantep, Turkey*For correspondence: eoguz73@yahoo.com 
STZ-induced diabetic rats exposed to IR (Kurcer et al., 2007). In our previous study we found that Mel treatment reversed the increase of serum tumor necrosis factor-alpha (TNF- $\alpha$ ) levels and histopathological injury in renal tissue after renal IR (Oguz et al., 2015). It has been reported that Mel has the potential to reduce MTX induced oxidative stress, neutrophil infiltration as well as renal damage at a dose of $40 \mathrm{mg} / \mathrm{kg}$ in rats (Abraham, 2010).

To the best of our knowledge there is not any study investigating the effect of Lyc on renal tissue after Mtx administration and the role of ceruloplasmin (CP) in Mtx induced nephrotoxicity. In this study we aimed to investigate the effect of Lyc alone or combined with Mel on renal damage, after Mtx administration by evaluating both biochemical and histopathological parameters and to investigate the role of CP in Mtx induced renal damage..

\section{Materials and Methods}

\section{Experimental protocol}

We used 26 healthy adult male Sprague-Dawley rats (weighing between 200-275g) in this study. Dollvet Animal Care and Use Committee has been approved the study. All experimental procedures were conducted in accordance with the Guide to the Care and Use of Laboratory Animals.

The animals were obtained from Dollvet Animal Labrotory (Sanliurfa,Turkey) where the experimental protocole was performed. The animals were allowed to acclimate under standard laboratory conditions (12-h light:12-h dark) ) in a room with controlled temperature $\left(24 \pm 3^{\circ} \mathrm{C}\right) 1$ week prior to the experimental study. The animals had free access to water and were fed a standard commercial pellet diet ad libitum.

After 7 days of acclimatization, the animals were divided into four groups.

Group 1 (6 rats); Vehicle was administrated to the rats in this group and served as control.

Group 2 (6 rats ); Rats were injected intraperitoneally (i.p.) with a single dose of $20 \mathrm{mg} / \mathrm{kg}$ of Mtx (Avadi et al., 2011; Bozkurt et al., 2014) and given corn oil by oral gavage (o.g).

Group 3 (7 rats ); Rats were treated with Lyc in corn oil (10 mg/kg, o.g.) (Augusti et al., 2007) for 10 days after a single dose of MTX (20 mg/kg, i.p.).

Group 4 ( 7 rats ); Rats were treated with Lyc in corn oil (10 mg/kg, o.g.) and Mel (40 mg/kg, i.p.) (Abraham et al., 2010) for 10 days after a single dose of MTX (20 $\mathrm{mg} / \mathrm{kg}$, i.p.). Mel was prepared by dissolving $50 \mathrm{mg}$ in 2 $\mathrm{ml}$ ethanol and diluted to $100 \mathrm{ml}$ with distilled water to make $0.2 \%$ solution (Mohamed et al., 2005).

All rats were sacrificed and kidneys were removed immediately, decapsulated, and divided longitudinally into two equivalent sections. One of the sections were fixed with $10 \%$ buffered formalin solution at room temperature for histopathological evaluation and the other one was stored at $-80{ }^{\circ} \mathrm{C}$ for biochemical analysis.

\section{Histopathological examination}

Renal tissue samples were fixed in $10 \%$ neutral buffered formalin, embedded in paraffin, sections of 4 $\mu \mathrm{m}$ were cut, and stained with hematoxylin and eosin for histological examination using light microscopy (Olympus BX51 TF Tokyo Japan) by a pathologist who was blinded to the study groups.

Renal injury has been described as mentioned by Bozkurt et al. (2012). Tubular degeneration, tubular dilatation, tubular cell swelling and tubular architectural impairment, was scored semi-quantitatively using three slides from each kidney specimen at at $\times 200$ objectives magnification to determine renal injury of the cortex and medulla. The score was described as: normal, 0; involvement of $\leq 10 \%$ of the cortex and medulla, 1 ; involvement of $11-20 \%$ of the cortex and medulla, 2; involvement of $21-30 \%$ of the cortex and medulla, 3 ; involvement of $>30 \%$ of the cortex and medulla.

Mononuclear inflammatory cells in the interstitium were scored as follows: no accumulation, 0; slight accumulation, 1; moderate accumulation, 2; extensive accumulation, 3 .

\section{Biochemical measurements}

Measurement of tissue TNF- $\alpha$, interleukin 1-beta (IL-1 $\beta$ ) and nitric oxide (NO)

The concentration of tissue TNF- $\alpha$, IL- $1 \beta$ and NO were measured by using a commercially available rat TNF- $\alpha$ enzyme-linked immunosorbent assay (ELISA) kit (Ray Biotech Inc., Diaclone, Cayman respectively).

\section{Measurement of tissue $C P$}

The enzymatic activity of CP was measured according to Erel's method (Erel, 1998). Using this assay, ferrous ion is oxidized to ferric ion via $\mathrm{CP}$ ferroxidase activity. The results are expressed as units per gram protein.

\section{Statistical analyses}

The results were reported as the means \pm standard deviation for biochemical measurements and as minmax for histopathological measurements. Data analyses were performed using the statistical software SPSS for Windows (version). The Kruskal-Wallis test was used to compare the groups for both histopathologic and biochemical parameters. A Bonferroni-adjusted MannWhitney U test was used to compare the two groups. The values of less than 0.05 were regarded as statistically significant.

\section{Results}

The renal tissues of the control group were with no pathological change and had normal structures according to histopathological evaluation. Marked degenerative changes such as tubular degeneration, tubular dilatation, tubular cell swelling, and tubular architectural impairment were obserrved in Mtx group. Treatment both with Lyc and Lyc + mel provided an obvious improvement in the degenerative changes compared to Mtx administrated group (Figure 1, Table 1.). In Lyc treated group, a significant improvement was seen in tubular degeneration $(\mathrm{p}<0.01)$. In Lyc+Mel group there was also a significant improvement in tubular dilatation and tubular cell swelling compared to Mtx group $(\mathrm{p}<0.01)$ (Table 1). 
Table 1. Histopathologic Examination Results in rat Renal Tissues

\begin{tabular}{lcccc}
\hline Histopathologic Parameters & Control & Mtx & Mtx+Lyc & Mtx+Lyc+Mel \\
\hline Tubular degeneration & $(0.0-1.0)$ & $(1.0-3.0)^{\mathrm{a}}$ & $(0.0-2.0)^{\mathrm{b}}$ & $(0.0-1.0)^{\mathrm{b}}$ \\
Tubular dilatation & $(0.0-1.0)$ & $(1.0-2.0)^{\mathrm{a}}$ & $(0.0-1.0)$ & $(0.0-1.0)^{\mathrm{b}}$ \\
Tubular cell swelling & $(0.0-1.0)$ & $(1.0-2.0)^{\mathrm{a}}$ & $(0.0-2.0)$ & $(0.0-1.0)^{\mathrm{b}}$ \\
Tubular architectural impairment & $(0.0-1.0)$ & $(0.0-1.0)$ & $(0.0-1.0)$ & $(0.0-1.0)$ \\
\hline
\end{tabular}

*Mtx: methotrexate, Lyc: lycopene, Mel: melatonin; Data were expressed as (min-max); ${ }^{\mathrm{a}, \mathrm{b}}$ Significantly different from Control and Mtx group respectively $(\mathrm{p}<0.01)$

Table 2. Biochemical Parameters Measured in the Renal Tissues of Rats

\begin{tabular}{lcccr}
\hline Biochemical Parameters & Control & Mtx & Mtx+Lyc & Mtx+Lyc+Mel 1 \\
\hline TNF- $\alpha$ (pg/gr protein) & $2302.19 \pm 126.39$ & $3164.75 \pm 223.99^{\mathrm{a}}$ & $2524.92 \pm 324.92^{\mathrm{b}}$ & $2660.20 \pm 267.83^{\mathrm{b}}$ \\
IL-1 $\beta$ (pg/gr protein) & $62.34 \pm 2.31$ & $81.29 \pm 7.25^{\mathrm{a}}$ & $63.09 \pm 9.84^{\mathrm{b}}$ & $63.18 \pm 9.63^{\mathrm{b}}$ \\
NO $(\mu$ molar/gr protein $)$ & $5.17 \pm 0.63$ & $8.43 \pm 1.40^{\mathrm{a}}$ & $7.69 \pm 0.99$ & $5.38 \pm 0.38^{\mathrm{b}}$ \\
CP $($ U/gr protein) & $54.16 \pm 1.45$ & $61.39 \pm 2.86^{\mathrm{a}}$ & $54.35 \pm 3.26^{\mathrm{b}}$ & $53.55 \pm 2.57^{\mathrm{b}}$ \\
\hline
\end{tabular}

*TNF- $\alpha$ :Tumor Necrosis Factor-alpha, IL-1 $\beta$ :Interleukin -1 beta, NO: Nitric oxide, CP: ceruloplasmin; Data are expressed as Mean \pm SD;

${ }^{\mathrm{a}, \mathrm{b}}$ Significantly different from Control and Mtx group respectively $(\mathrm{p}<0.01)$

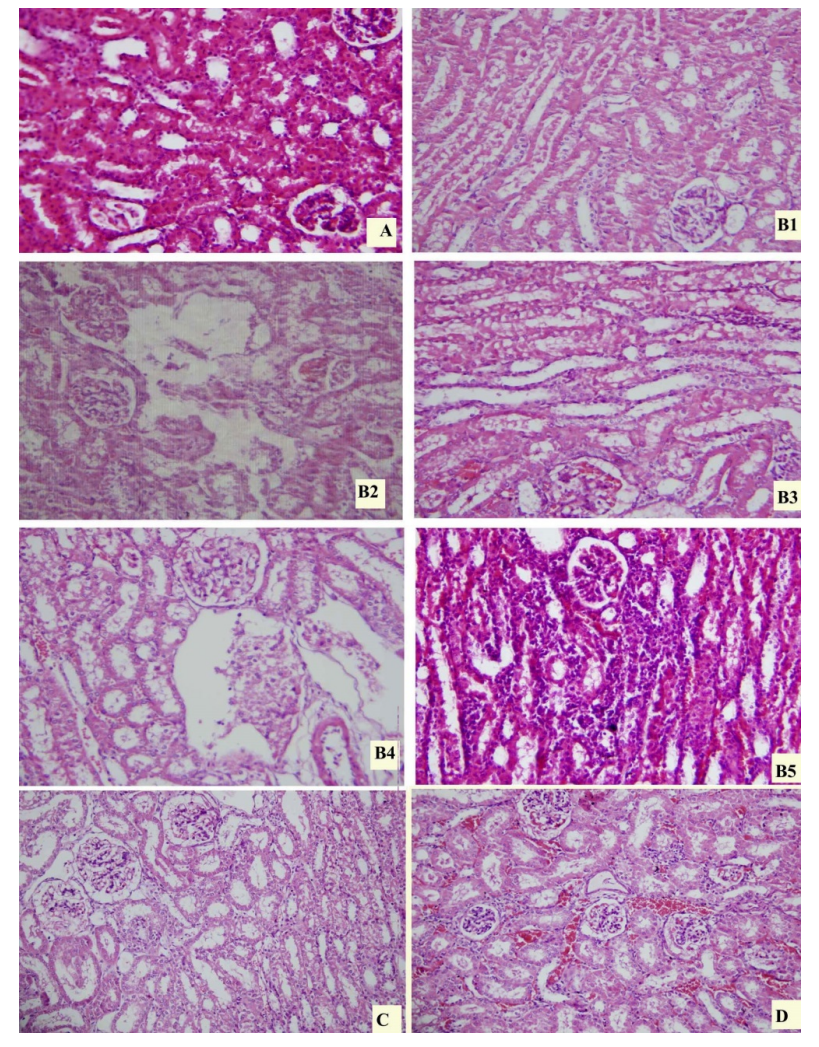

Figure 1. Histopathological Section of Renal Tissues (Hematoxylin Eosin Staining x200 Objectives). A) Control group; normal histological appearence of HE stained renal tissues. B1-5) Mtx group; B1) tubular degeneration, B2) tubular dilatation, B3) Tubular cell swelling, B4) tubular architectural impairment, B5) Inflammatory cell infiltration. C) Lyc treated group; Improvement in tubular structure compared with MTX group. D) Lyc+Mel treated group; Improvement in tubula rstructure and minimal cellular swelling compared with MTX group

The biochemical parameters indicating the effects of Lyc alone or Lyc+Mel on renal tissue against Mtxintoxicated rats are shown in Table 2. TNF- $\alpha$ and IL-1 $\beta$ levels of renal tissue were increased significantlay in Mtx group compared to control. A significant reduction was observed in both Lyc and Lyc+Mel treatment compared to Mtx group in these proinflammatory cytokines $(\mathrm{p}<0.01)$. There was no significant difference between Lyc and Lyc + Mel treated groups in terms of TNF- $\alpha$ and IL- $1 \beta$ levels . A significant reduction was observed in both Lyc and Lyc and Mel combined treatment compared to Mtx group in tissue CP levels $(\mathrm{p}<0.01)$. The decrease in CP level was more in Lyc and Mel combined group, but it was not significant. There was a significant increase in NO levels of renal tissue in Mtx administrated group. The reduction of NO levels was not significant in the Lyc treated group compared to Mtx group. Hovewer, there was a significant decrease in Lyc + Mel treatment group compared to Mtx group $(\mathrm{p}<0.01)$.

\section{Discussion}

Nephrotoxicity remains a vexing complication of chemotherapeutic agents. A number of kidney lesions can result from these drugs, including primarily tubularlimited dysfunction, glomerular injury with proteinuria, full-blown acute kidney injury, and long-term chronic kidney injury. Nephrotoxicity is an important side-effect of treatment with Mtx since more than $90 \%$ of Mtx is cleared by the kidneys and the clearence of Mtx delays. Consequently, elevated plasma Mtx concentration can increasethe risk of other systemic toxicities and Mtx -induced renal toxicity can be life threatening (Abelson et al., 1983; Lameire et al., 2011).

It was reported that $20 \mathrm{mg} / \mathrm{kg}$ dose of Mtx, used in this study, highly exceeds interval dose and can be accepted as high dose Mtx in human (Oktem et al., 2006; Cetinkaya et al., 2007; Abdel-Raheem and Khedr, 2014). Mtx treatment at high doses may cause renal failure (Abelson et al., 1983). Hovewer, glomerular function and tubular function are reported to be altered after low-dose Mtx therapy in patients with rheumatoid arthritis (Fiehn, 2011).

In this study, we used Mel at a dose of $40 \mathrm{mg} / \mathrm{kg}$ since it was reported that Mel has the potential to reduce Mtx induced oxidative stress, neutrophil infiltration as well 
as renal damage at this dose (Abraham et al., 2010). Lyc was used at adose of $10 \mathrm{mg} / \mathrm{kg}$ since it was showed that it attenuates cyclosporine-induced renal dysfunction and oxidative stress in rats at this dose (Atessahin et al., 2007).

We observed that Mtx administration caused significant tubular degeneration, tubular dilatation and tubular cell swelling in renal tissues. Lyc treatment provided improvement in tubular degeneration, tubular dilatation and tubular cell swelling, but only the improvement in tubular degeneration was significant $(\mathrm{p}<0.05)$. Hovewer, Lyc and Mel combined therapy provided a significant improvement in all histopathological changes including tubular degeneration, tubular dilatation and tubular cell swelling $(\mathrm{p}<0.05)$ These data indicate Lyc may provide improvement in Mtx induced renal toxicity, but the improvement with Lyc and Mel combination provides more benefical effect compared to alone Lyc treatment.

The pathogenesis of Mtx nephrotoxicity involves multiple pathways, including oxidative stress and inflammation (Devrim et al., 2005; Asvadi et al., 2011). Inflammatory mediators including TNF- $\alpha, \mathrm{IL}-1$ and NO, known as endothelium-derived relaxing factor (EDRF) are shown to play important roles in the pathogenesis of Mtx nephrotoxicity (Ibrahim et al., 2014; Kirbas et al., 2015).

$\mathrm{NO}$, is an important cellular signaling molecule, involved in many physiological and pathological processes (Hou et al., 1999). It is reported that nitric oxide is involved in the acute renal failure since free radical nature of nitric oxide might contribute to tubular damage (Christo et al., 2011). It has been stated that iNOS can be found in extremely low amounts or not at all in normal renal tissue but increases in nephropathy (Manikandan et al., 2011). Uz et al. (2005) found that the NO level was increased in the kidney with MTX, indicating that MTX-induced renal damage may be mediated by high NO. Ibrahim et al. (2014) also reported that MTX caused renal oxidative/nitrosative stress, indicated by decrease in GSH and catalase with increase in malondialdehyde and nitric oxide (NOx) level (Ibrahim et al., 2014). Similar to these reports, we also found that $\mathrm{NO}$ levels are increased in renal tissue of Mtx administrated rats.

The studies reveal that cytokines such as interleukin (IL) -1 and TNF- $\alpha$ are secreted after infection, trauma and hypoxia. These proinflammatory cytokines release large amounts of toxic reactive oxygen species and cause cellular injury by activating inflammatory cells (Ramesh and Reeves, 2002). The studies concerned that TNF and IL play role in Mtx induced nephrotoxicity (Morsy et al., 2013; Kirbas et al., 2015). In our study we also found that MTX administration caused a significant increase in the levels of TNF- $\alpha$ and IL- 1 in renal tissue, indicating the role of these cytokines in MTX-induced nephrotoxicity.

$\mathrm{CP}$ is an acute-phase reactant that is synthesized and secreted by the liver as well as monocyte/macrophages, and it participates in both iron and copper metabolism (Fox et al., 2000). CP is a major hypoxia-activated systemic NO oxidase that seems to be able to function in both antioxidant and pro-oxidant capacities (Fox et al., 1995). It was reported that $\mathrm{CP}$ may be a potential diagnostic biomarker for diabetic nephropathy (Ito et al., 2008) and new potential urinary biomarker (Wang et al., 2013). In this study, CP levels of the renal tissues were increased significantly in Mtx administrated group compared to control. To the best of our knowledge there is not any study revealing the role of $\mathrm{CP}$ in Mtx induced nephrotoxicity. In this study we firstly found that CP may have a role in Mtx induced nephrotoxicity.

We evaluated both the effect of Lyc and Lyc+Mel treatment in Mtx induced nephrotoxicity. Lyc treatment decreased the NO levels but it was not significant. Hovewer, Lyc and Mel combined treatment decreased the NO levels significantly compared to Mtx administrated group. Both Lyc alone and Lyc and Mel combined therapy decreased TNF- $\alpha$, IL-1 $\beta$ and CP levels of renal tissue compared to Mtx intoxicated rats. There was no difference in terms of these biomarkers between Lyc alone and Lyc and Mel combined groups.

Both pathologic improvements in histopathological changes by a significance in tubular degeneration and significant reduction in TNF- $\alpha$, IL-1 $\beta$ and CP levels shows that Lyc may have benefical effects on Mtx induced renal damage. Additionally, a significant decrease in NO levels and in all histopathological changes including tubular degeneration, tubular dilatation and tubular cell swelling by Lyc and Mel treatment indicate that Lyc and Mel showed more potential effect than Lyc alone on Mtx induced nephrotoxicity.

Several reports clearly indicated that the Lyc which possesses many conjugated double bonds is known with its high antioxidant activity and free radical scavenging capacity (Cohen, 2002; Jonker et al., 2003; Michael McClain and Bausch, 2003; Tapiero et al., 2004; Velmurugan et al., 2004; Rostoka 2010). Lyc treatment, either before or after cisplatin administration, provided a significant protection against cisplatin-induced nephrotoxicity (Atessahin et al., 2005). The histological evaluation of the kidney preparations in pretreatment group also revealed a decrease cisplatin-induced tubular necrosis. Palabiyik et al. (2013) reported that Lyc might be partially protective against ochratoxin A-induced nephrotoxicity and oxidative stress in rat as evidenced by partial recovery in histopathology, apoptosis and antioxidant parameters.

Lyc may be beneficial in the prevention and treatment of oxidative stress and inflammation in the kidney due to obesity. It reduces TNF- $\alpha$ via the receptor of advanced glycation end products which is a member of the immunoglobulin superfamily (Pierine et al., 2014). Lyc significantly improved oxidative stress, inflammation, lipid peroxidation, autophagy, and apoptosis in rats with contrast induced nephrotoxicity (Buyuklu et al., 2015). Mel reduced the oxidative burden, attenuated the chronic inflammation and limited apoptosis. In several animal models of chronic kidney disease, involving experimental hypertension, diabetes mellitus and various models of nephrotoxicity (Hrenak et al., 2015). Mel treatment reversed the increase of serum TNF- $\alpha$ levels and histopathological injury in renal tissue after renal IR (Oguz et al., 2015). Mel, with a dose of $40 \mathrm{mg} / \mathrm{kg}$, has the potential to reduce MTX induced oxidative stress, neutrophil infiltration as well as renal damage (Abraham et al., 2010). 
There are also several studies revealing that combination of antioxidants including Lyc, Mel and the the others may be more benefical in some pathological conditions. The combined effect of erythropoietin (EPO) plus Mel, which are known anti-inflammatory and antioxidant agents, were determined in IR-induced renal injury in rats. EPO and MEL combination treatment exerted more nephroprotective effects than EPO treatment (Ahmadiasl, 2014).

Vitamin 1,25-dihydroxyvitamin D3 (VD3) combined with Mel significantly reduced apoptotic and histological alterations when compared with Mel or VD3 alone after ischemia-reperfusion injury in the rat kidney (Sinanoglu et al., 2012). The combined treatment with Lyc and Mel showed potential reduction in increased nitric oxide (NO) and malondialdehyde (MDA) induced by cisplatin administration more than those treated with lyco alone (Al-Malki, 2014). Moselhy and Al mslmani (2008) reported that Lyc when combined with Mel may be useful for the prevention of breast cancer by inhibition of lipid peroxidation.

We suggest that Lyc is effective in the tretament of Mtx induced renal damage by decreasing the increased proinflammatory cytokines and also increased CP levels which was firstly showed in this study. The combination of Mel and Lyc may be an alternative therapy since has more benefical effects in histopathological improvement and in $\mathrm{NO}$ levels. However, further experimental and clinical studies studies are required to reveal the exact mechanisms mediating the effect of Lyc and Mel combination therapy in Mtx induced renal damage.

\section{References}

Abdel-Raheem T, Khedr NF (2014). Renoprotective effects of montelukast, a cysteinyl leukotriene receptor antagonist, against methotrexate-induced kidney damage in rats. Naunyn Schmiedebergs Arch Pharmacol, 387, 341-53.

Abelson HT, Fosburg MT, Beardsley GP, et al (1983). Methotrexate-induced renal impairment: clinical studies and rescue from systemic toxicity with high-dose leucovorin and thymidine. J Clin Oncol, 1, 208-16.

Abraham P, Kolli VK, Rabi S (2010). Melatonin attenuates methotrexate-induced oxidative stress and renal damage in rats. Cell Biochem Funct, 28, 426-33.

Ahmadiasl N, Banaei S, Alihemati A, Baradaran B, Azimian E (2014). Effect of a combined treatment with erythropoietin and melatonin on renal ischemia reperfusion injury in male rats. Clin Exp Nephrol, 18, 855-64.

Al-Malki AL (2014). Synergestic effect of lycopene and melatonin against the genesis of oxidative stress induced by cyclophosphamide in rats. Toxicol Ind Health, 30,570-5.

Asvadi I, Hajipour B, Asvadi A, et al (2011). Protective effect of pentoxyfilline in renal toxicity after methotrexate administration. Eur Rev Med Pharmacol Sci, 15, 1003-9.

Atessahin A, Ceribasi AO, Yilmaz S (2007). Lycopene, a carotenoid, attenuates cyclosporine-induced renal dysfunction and oxidative stress in rats. Basic Clin Pharmacol Toxicol, 100, 372-6.

Atessahin A, Yilmaz S, Karahan I, Ceribasi AO, Karaoglu A (2005). Effects of lycopene against cisplatin-induced nephrotoxicity and oxidative stress in rats. Toxicology, 212, 116-23.
Augusti PR, Conterato GM, Somacal S, et al (2007). Effect of lycopene on nephrotoxicity induced by mercuric chloride in rats. Basic Clin Pharmacol Toxicol, 100, 398-402.

Baydas G, Canatan H, Turkoglu A (2002). Comparative analysis of the protective effects of melatonin and vitamin $\mathrm{E}$ on streptozocin-induced diabetes mellitus. J Pineal Res, 32, 225-30.

Bozkurt M, Em S, Oktayoglu P, et al (2014). Carvacrol prevents methotrexate-induced renal oxidative injury and renal damage in rats. Clin Invest Med, 37, 19-25.

Buyuklu M, Kandemir FM, Ozkaraca M, et al (2015). Benefical effects of lycopene against contrast medium-induced oxidative stress, inflammation, autophagy, and apoptosis in rat kidney. Hum Exp Toxicol, 34, 487-96.

Cetinkaya A, Kurutas EB, Bulbuloglu E, Kantarceken B (2007). The effects of $\mathrm{N}$-acetylcysteine on methotrexate-induced oxidative renal damage in rats. Nephrol Dial Transplant, 22, 284-5.

Choudhury RC, Ghosh SK, Palo AK (2000). Cytogenetic toxicity of methotrexate in mouse bone marrow. Environ Toxicol Pharmacol, 3, 191-6.

Christo JS, Rodrigues AM, Mouro MG, et al (2011). Nitric oxide (NO) is associated with gentamicin (GENTA) nephrotoxicity and the renal function recovery after suspension of GENTA treatment in rats. Nitric Oxide, 24, 77-83.

Clinton SK (1998). Lycopene: chemistry, biology, and implications for human health and disease. Nutr Rev, 56, 35-51.

Cohen LA (2002). A review of animal model studies of tomato carotenoids, lycopene, and cancer chemoprevention. Exp Biol Med, 227, 864-8.

D'Adamo DR (2011). Appraising the current role of chemotherapy for the treatment of sarcoma. Seminars in Oncology, 38, 19-9.

Devrim E, Cetin R, Kilicoglu B, et al (2005). Methotrexate causes oxidative stress in rat kidney tissues. Ren Fail, 27, 771-3.

Di Mascio P, Kaiser S, Sies H (1989). Lycopene as the most efficient biological carotenoid singlet oxygen quencher. Arch Biochem Biophys, 274, 532-8.

Erel O (1998). Automated measurement of serum ferroxidase activity. Clin Chem, 44, 2313-9.

Fiehn C (2011). The other opinion: nephrotoxicity of low-dose methotrexate-a problem which does not exist].Z Rheumatol, 70, 825-6.

Findlay M, von Minckwitz G, Wardley A (2008). Effective oral chemotherapy for breast cancer: pillars of strength. Ann Oncol, 19, 212-22.

Forrest CM, Mackay GM, Stoy N, Stone TW, Darlington LG (2007). Inflammatory status and kynurenine metabolism in rheumatoid arthritis treated with melatonin. $\mathrm{Br} \mathrm{J} \mathrm{Clin}$ Pharmacol, 64, 517-526.

Fox PL, Mazumder B, Ehrenwald E, Mukhopadhyay CK (2000). Ceruloplasmin and cardiovascular disease. Free Radic Biol Med, 28, 1735-44.

Fox PL, Mukhopadhyay C, Ehrenwald E (1995). Structure, oxidant activity, and cardiovascular mechanisms of human ceruloplasmin. Life Sci, 56, 1749-58.

Gisondi P, Girolomoni G (2007). Biologic therapies in psoriasis: a new therapeutic approach. Autoimmun Rev, 6, 515-9.

Gultekin F, Delibas N, Yasar S, Kilinc I (2001). In vivo changes in antioxidant systems and protective role of melatonin and a combination of vitamin $C$ and vitamin $E$ on oxidative damage in erythrocytes induced by chlorpyrifos-ethyl in rats. Arch Toxicol, 75, 88-96.

Hou YC, Janzuk A, Wang PG (1999). Current trends inmthe development of nitric oxide donors. Curr Pharm Des, 5 , 


$$
\text { 417-41. }
$$

Hrenak J, Paulis L, Repova K, et al (2015). Melatonin and renal protection: novel perspectives from animal experiments and human studies (review). Curr Pharm Des, 21, 936-49.

Ibrahim MA, El-Sheikh AA, Khalaf HM, Abdelrahman AM (2014). Protective effect of peroxisome proliferator activator receptor (PPAR)- $\alpha$ and $-\gamma$ ligands againstmethotrexateinduced nephrotoxicity. Immunopharmacol Immunotoxicol, 36, 130-7.

Ito H, Fujita H, Takahashi T (2008). Diagnostic biomarkers of diabetic nephropathy. Expert Opin Med Diagn, 2, 61-9.

Kedziora-Kornatowska K (2008). Antioxidative effects of melatonin administration in elderly primary essential hypertension patients. J Pineal Res, 45, 312-7.

Kirbas A, Cure MC, Kalkan Y, et al (2015). Effect of infliximab on renal injury due to methotrexate in rat. Iran J Kidney Dis, 9, 221-9.

Koul A, Shubrant, Gupta P (2013). Phytomodulatory potential of lycopene from Lycopersicum esculentum against doxorubicin induced nephrotoxicity. Indian J Exp Biol, 51, 635-45.

Kurcer Z, Parlakpinar H, Vardi N, et al (2007). Protective effects of chronic melatonin treatment against renal ischemia/ reperfusion injury in streptozotocin-induced diabetic rats. Exp Clin Endocrinol Diabetes, 115, 365-71.

Lameire N, Kruse V, Rottey S (2011). Nephrotoxicity of anticancer drugs-an underestimated problem. Acta Clin Belg, 66, 337-45.

Li W, Wang G, Lu X, et al (2014). Lycopene ameliorates renal function in rats with streptozotocin-induced diabetes. Int $J$ Clin Exp Pathol, 7, 5008-15.

Manikandan R, Beulaja M, Thiagarajan R, et al (2011). Ameliorative effects of curcumin against renal injuries mediated by inducible nitric oxide synthase and nuclear factor kappa B during gentamicin-induced toxicity in Wistar rats. Eur J Pharmacol, 30, 578-85.

Miyazaki J, Kawai K, Hayashi H, et al (2003). The limited efficacy of methotrexate, actinomycin D and cisplatin (MAP) for patients with advanced testicular cancer. Jpn J Clin Oncol, 33, 391-5.

Mohamed AM, Hosny AH, Mahmoud HM, et al (2005). The biochemical and morphological alterations following administration of melatonin, retinoic acid and nigella sativa in mammary carcinoma: an animal model. Int J Exp Pathol, 86, 383-96.

Mordente A, Guantario B, Meucci E, et al (2011). Lycopene and cardiovascular diseases: an update. Curr Med Chem, 18, 1146-63.

Morsy MA, Ibrahim SA, Amin EF, et al (2013). Curcumin ameliorates methotrexate-induced nephrotoxicity in rats. Adv Pharmacol Sci, 387071.

Moselhy SS, Al mslmani MA (2008).Chemopreventive effect of lycopene alone or with melatonin against the genesis of oxidative stress and mammary tumors induced by 7,12 dimethyl(a)benzanthracene in sprague dawely female rats. Mol Cell Biochem, 319, 175-80.

Oguz E, Yilmaz Z, Ozbilge H(2015). Effects of melatonin on the serum levels of pro-inflammatory cytokines and tissue injury after renal ischemia reperfusion in rats. Ren Fail, 37, 318-22.

Oktem F, Yilmaz HR, Ozguner F, et al (2006). Methotrexateinduced renal oxidative stress in rats: the role of a novel antioxidant caffeic acid phenethyl ester. Toxicol Ind Health, 22, 241-7.

Palabiyik SS, Erkekoglu P, Zeybek ND, et al (2013). Protective effect of lycopene against ochratoxin $\mathrm{A}$ induced renal oxidative stress and apoptosis in rats. Exp Toxicol Pathol, $\mathbf{6 5}, 853-61$.
Peters BP, Weissman FG, Gill MA. Pathophysiology and treatment of psoriasis. Am J Health Syst Pharm, 57, 645-59.

Pierine DT, Navarro ME, Minatel IO, et al (2014). Lycopene supplementation reduces TNF- $\alpha$ via RAGE in the kidney of obese rats. Nutr Diabetes, $\mathbf{4}, 142$.

Ramesh G, Reeves WB (2002). TNF-alpha mediates chemokine and cytokine expression and renal injury in cisplatin nephrotoxicity. J Clin Invest, 110, 835-42.

Renna S, Cottone M, Orlando A (2014). Optimization of the treatment with immunosuppressants and biologics in inflammatory bowel disease. World J Gastroenterol, 20, 9675-90.

Rostoka E, Isajevs S, Baumane L, et al (2010). Effects of lycopene, indole-3-carbinol, and luteolin on nitric oxide production and iNOS expression are organ-specific in rats. Arh Hig Rada Toksikol, 61, 275-85.

Seigers R, Schagen SB, Beerling W, et al (2008). Long-lasting suppression of hippocampal cell proliferation and impaired cognitive performance bymethotrexate in the rat. Behav Brain Res, 186, 168-75.

Sinanoglu O, Sezgin G, Ozturk G, et al (2012). Melatonin with 1,25-dihydroxyvitamin D3 protects against apoptotic ischemia-reperfusion injury in the rat kidney. Ren Fail, 34, 1021-6.

Tapiero H, Townsend DM, Tew KD (2004). The role of carotenoids in the prevention of human pathologies. Biomed Pharmacother, 58, 100-10.

Uz E, Oktem F, Yilmaz HR, Uzar E, Ozgüner F (2005). The activities of purine-catabolizing enzymes and the level of nitric oxide in rat kidneys subjected tomethotrexate: protective effect of caffeic acid phenethyl ester. Mol Cell Biochem, 277, 165-70.

Velmurugan B, Santhiya ST, Nagini S (2004). Protective effect of S-allylcysteine and lycopene in combination against $\mathrm{N}$-methyl-N'-nitro-N-nitrosoguanidine-induced genotoxicity. Pol J Pharmacol, 56, 241-5.

Wang C, Li C, Gong W, Lou T (2013). New urinary biomarkers for diabetic kidney disease. Biomark Res, 1, 9 . 